\title{
Chaos in some planar nonautonomous polynomial differential equation
}

\author{
by KLAudiusz WóJcik (Kraków)
}

\begin{abstract}
We show that under some assumptions on the function $f$ the system $\dot{z}=\bar{z}\left(f(z) e^{i \phi t}+e^{i 2 \phi t}\right)$ generates chaotic dynamics for sufficiently small parameter $\phi$. We use the topological method based on the Lefschetz fixed point theorem and the Ważewski retract theorem.
\end{abstract}

1. Introduction. In this note we prove that the nonautonomous periodic system on the plane

$$
\dot{z}=\bar{z}\left(f(z) e^{i \phi t}+e^{i 2 \phi t}\right)
$$

generates chaotic dynamics for sufficiently small parameter $\phi$ (under some assumptions on the function $f$ ). The existence of a nonzero $2 \pi / \phi$-periodic solution of this system in case $\phi \neq 0$ follows from [S]. The problem of existence of periodic solutions of planar polynomial differential equations with periodic coefficients was also studied in [S1], [Ma], [MMZ]. The method presented in [S], [S1] is based on the Lefschetz Fixed Point Theorem and the Ważewski Retract Theorem. The results in [Ma] use classical topological degree techniques and a simple a priori bounds argument. Our result follows by the topological method for detecting chaotic dynamics introduced in [SW] and improved in [W1], [W2]. By chaos we mean the existence of a compact invariant set such that the Poincaré map is semiconjugate to the shift on two symbols and the counterimage (by the semiconjugacy) of any periodic point in the shift contains a periodic point of the Poincaré map. A similar definition of chaos was used in [MM] (compare also [W]). In order to apply this method we have to show the existence some sets, called periodic isolating segments. In all practical applications isolating segments are manifolds with corners. A basic property of such a segment is that at any point on the

2000 Mathematics Subject Classification: 37C25, 34C25.

Key words and phrases: periodic solutions, chaos, fixed point index, Lefschetz number. Research partially supported by the KBN grant 2 P03A 04010 and Foundation for Polish Science. 
boundary of the segment the vector field is directed either outward or inward with respect to the segment. We will impose only topological conditions on the segments and their exit sets and we do not require any information on the particular solution inside the segments.

2. Chaotic systems. Consider the system

$$
\dot{z}=\left(f(z) e^{i \phi t}+e^{i 2 \phi t}\right) \bar{z} .
$$

Assume that:
(A) $f: \mathbb{C} \rightarrow[0, \infty)$ is continuous,
(B) $f(0)=0$,
(C) $\lim _{|z| \rightarrow \infty} f(z)=\infty$.

We denote by $\Phi$ the local flow on $\mathbb{R} \times \mathbb{R}^{2}$ generated by the system (1), i.e. $\Phi_{\tau}\left(t_{0}, x_{0}\right)=\left(t_{0}+\tau, \varphi_{\left(t_{0}, \tau\right)}\left(x_{0}\right)\right)$, where $\varphi_{\left(t_{0}, \tau\right)}\left(x_{0}\right)$ is the value of the solution of the Cauchy problem associated with (1) at time $t_{0}+\tau$. The map $\varphi$ is called the local process generated by (1) (cf. [S]). It is continuous with respect to $\left(t_{0}, \tau, x_{0}\right)$ and satisfies

$$
\begin{aligned}
\varphi_{(\sigma, 0)} & =\mathrm{id}_{\mathbb{R}^{2}}, \\
\varphi_{(\sigma, t+s)} & =\varphi_{(\sigma+t, s)} \circ \varphi_{(\sigma, t)}, \\
\varphi_{(\sigma+T, t)} & =\varphi_{(\sigma, t)}, \quad T=2 \pi / \phi .
\end{aligned}
$$

In order to determine all $T$-periodic solutions of (1) it suffices to look for the fixed points of $\varphi_{(0, T)}$ (the latter function is called the Poincaré map).

Let $\Sigma_{2}$ denote the set of bi-infinite sequences of two symbols and let $\sigma$ be the shift map on $\Sigma_{2}$ (see [W]). We say that the equation (1) is $\Sigma_{2}$-chaotic if there exist a compact set $I$ invariant with respect to the Poincaré map $\varphi_{(0, T)}$ and a continuous surjective map $g: I \rightarrow \Sigma_{2}$ such that:

(j) $\sigma \circ g=g \circ \varphi_{(0, T)}$, i.e. $\varphi_{(0, T)}$ is semiconjugate to the shift map $\sigma$ : $\Sigma_{2} \rightarrow \Sigma_{2}$ in the set $I$,

(jj) for every $n$-periodic sequence $s \in \Sigma_{2}$ its counterimage $g^{-1}(s)$ contains at least one $n$-periodic point of $\varphi_{(0, T)}$.

It follows in particular that a $\Sigma_{2}$-chaotic equation has periodic solutions with primitive period $k T$ for every $k \in \mathbb{N}$ and the topological entropy of $\varphi(0, T)$ is positive.

REMARK 1. Since $I$ is compact and the set of periodic points is dense in $\Sigma_{2}$, the condition (jj) implies that the semiconjugacy $g$ must be a surjection.

The main result of this paper is the following

THEOREM 1. There is $\phi_{0}>0$ such that for all $0<\phi<\phi_{0}$ the equation (1) is $\Sigma_{2}$-chaotic. 
REMARK 2. Let

$$
\dot{z}=\left(f(z) e^{i k \phi t}+e^{i l \phi t}\right) \bar{z}
$$

where $f$ satisfies $(\mathrm{A}),(\mathrm{B}),(\mathrm{C})$ and $k, l \in \mathbb{N}$ are such that $k+l=1(\bmod 2)$. Using similar arguments to our proof of Theorem 1 one can check that this system also generates chaotic dynamics for sufficiently small $\phi$.

The proof of Theorem 1 will be presented in the last section. First we recall the notion of periodic isolating segments and the geometric method for detecting chaotic behaviour introduced in [SW] and improved in [W1], [W2].

3. Periodic isolating segments. Put $M=\mathbb{R} \times \mathbb{R}$. Let $f$ be a time dependent vector field on $M$. Consider the local process generated by the system

$$
\left\{\begin{array}{l}
\dot{t}=1, \\
\dot{x}=f(t, x) .
\end{array}\right.
$$

We let $\pi_{1}:[0, T] \times M \rightarrow[0, T]$ and $\pi_{2}:[0, T] \times M \rightarrow M$ denote the projections, and for $Z \subset \mathbb{R} \times M$ and $t \in \mathbb{R}$ we put $Z_{t}=\{x \in M:(t, x) \in Z\}$.

Let $\left(W, W^{-}\right)$be a pair of subsets of $[0, T] \times M$. We call $W$ a periodic isolating segment over $[0, T]$ (for the equation $(2)$ ) and $W^{-}$the exit set of $W$ if:

(i) $W$ and $W^{-}$are compact ENR's, $W_{0}=W_{T}$ and $W_{0}^{-}=W_{T}^{-}$,

(ii) there exists a homeomorphism

$$
h:[0, T] \times\left(W_{0}, W_{0}^{-}\right) \rightarrow\left(W, W^{-}\right)
$$

such that $\pi_{1}=\pi_{1} \circ h$,

(iii) for every $\sigma \in[0, T)$ and $x \in \partial W_{\sigma}$ there exists a $\delta>0$ such that for every $t \in(0, \delta)$ either $\varphi_{(\sigma, t)}(x) \notin W_{\sigma+t}$ or $\varphi_{(\sigma, t)}(x) \in \operatorname{int} W_{\sigma+t}$,

(iv) $W^{-} \cap([0, T) \times M)=\{(\sigma, x) \in W: \sigma<T, \exists \delta>0 \forall t \in(0, \delta)$ : $\left.\varphi_{(\sigma, t)}(x) \notin W_{\sigma+t}\right\}$.

The above definition introduced in $[\mathrm{SW}]$ is an inessential modification of the notion of periodic isolating block in $[\mathrm{S}]$. Notice that a $T$-periodic isolating block (in the sense of $[\mathrm{S}]$ ) can be easily obtained by gluing translated copies of periodic isolating segments over $[0, T]$.

Define a homeomorphism

$$
\widetilde{h}:\left(W_{0}, W_{0}^{-}\right) \rightarrow\left(W_{T}, W_{T}^{-}\right)=\left(W_{0}, W_{0}^{-}\right)
$$

by $\widetilde{h}(x)=\pi_{2}\left(h\left(T, \pi_{2} h^{-1}(0, x)\right)\right)$ for $x \in W_{0}$. Geometrically, $\widetilde{h}$ moves a point $x \in W_{0}$ to $W_{T}=W_{0}$ along the $\operatorname{arc} h\left([0, T] \times\left\{\pi_{2} h^{-1}(0, x)\right\}\right)$. A different choice of the homeomorphism $h$ in (ii) leads to a map which is homotopic to $\widetilde{h}$ (see $[\mathrm{S}]$ ), hence the automorphism

$$
\mu_{W}=\widetilde{h}_{*}: H\left(W_{0}, W_{0}^{-}\right) \rightarrow H\left(W_{0}, W_{0}^{-}\right)
$$


induced by $\widetilde{h}$ in singular homology is an invariant of the segment $W$. Recall that its Lefschetz number is defined as

$$
\operatorname{Lef}\left(\mu_{W}\right)=\sum_{n=0}^{\infty}(-1)^{n} \operatorname{tr} \widetilde{h}_{* n} .
$$

In particular, if $\mu_{W}=\mathrm{id}_{H\left(W_{0}, W_{0}^{-}\right)}$then $\operatorname{Lef}\left(\mu_{W}\right)$ is equal to the Euler characteristic $\chi\left(W_{0}, W_{0}^{-}\right)$

Let $Z, W$ be two periodic isolating segments over $[0, T]$ for the equation (2). Assume that:
(a) $Z \subset W, Z_{0}=W_{0}, Z_{0}^{-}=W_{0}^{-}$,
(b) $\mu_{Z}=\operatorname{id}_{H\left(W_{0}, W_{0}^{-}\right)}$.

The following result was proved in $[\mathrm{SW}]$ :

TheOREM 2. Assume that the conditions (a), (b) hold and moreover:

(1) $\mu_{W}^{2}=\operatorname{id}_{H\left(Z_{0}, Z_{0}^{-}\right)}$,

(2) $\operatorname{Lef}\left(\mu_{W}\right) \neq \chi\left(Z_{0}, Z_{0}^{-}\right)$

(3) $\chi\left(Z_{0}, Z_{0}^{-}\right) \neq 0$.

Then the equation (2) is $\Sigma_{2}$-chaotic.

4. Proof of Theorem 1. Our proof consists in the construction of two periodic isolating segments $Z$ and $W$ over $[0,2 \pi / \phi]$ satisfying the assumptions of Theorem 2. We describe briefly what the segments look like. The smaller segment $Z$ is a twisted prism with a square base centered at the origin. Its cross-sections $Z_{t}$ are obtained by rotating the base with angle velocity $\phi$ over the $t$-interval $[0,2 \pi / \phi]$. The exit set $Z^{-}$consists of two ribbons winding around the prism. The larger segment $W$ is also a twisted square based prism, but the sections $W_{t}$ are obtained by rotating the base with angle velocity $\phi / 2$ over the $t$-interval $[0,2 \pi / \phi]$. Its exit set $W^{-}$consists of two ribbons winding around the prism.

We denote by $F$ the vector field in the extended phase space $\mathbb{R}^{3}$ generated by the right-hand side of the system (1), i.e.

$$
F(t, x, y)=\left(\begin{array}{c}
1 \\
x f(z) \cos (\phi t)+y f(z) \sin (\phi t)+x \cos (2 \phi t)+y \sin (2 \phi t) \\
x f(z) \sin (\phi t)-y f(z) \cos (\phi t)+x \sin (2 \phi t)-y \cos (2 \phi t)
\end{array}\right) .
$$

Assume that $R>0$. Put

$$
\begin{aligned}
& \Lambda_{R}^{1}(t, x, y)=\frac{1}{R^{2}}(x \cos (\phi t / 2)+y \sin (\phi t / 2))^{2}-1, \\
& \Lambda_{R}^{2}(t, x, y)=\frac{1}{R^{2}}(x \sin (\phi t / 2)-y \cos (\phi t / 2))^{2}-1,
\end{aligned}
$$


and

$$
\begin{aligned}
& L_{R}=\left\{(t, x, y) \in \mathbb{R}^{3}: \Lambda_{R}^{i}(t, x, y) \leq 0, i=1,2\right\}, \\
& L_{R}^{-}=\left\{(t, x, y) \in \mathbb{R}^{3}: \Lambda_{R}^{1}(t, x, y)=0, \Lambda_{R}^{2}(t, x, y) \leq 0\right\}, \\
& L_{R}^{+}=\left\{(t, x, y) \in \mathbb{R}^{3}: \Lambda_{R}^{1}(t, x, y) \leq 0, \Lambda_{R}^{2}(t, x, y)=0\right\} .
\end{aligned}
$$

LEMma 1. If $\phi>0$ is sufficiently small and $R$ is large enough then

$$
\begin{array}{ll}
F(t, x, y) \cdot \nabla \Lambda_{R}^{1}(t, x, y)>0 & \left((t, x, y) \in L_{R}^{-}\right), \\
F(t, x, y) \cdot \nabla \Lambda_{R}^{2}(t, x, y)<0 & \left((t, x, y) \in L_{R}^{+}\right) .
\end{array}
$$

P r o of. A direct calculation shows that

(5)

$F(t, x, y) \cdot \nabla \Lambda_{R}^{1}(t, x, y)=\frac{2}{R^{2}}(x \cos (\phi t / 2)+y \sin (\phi t / 2))$

$\times(f(z)(x \cos (\phi t / 2)+y \sin (\phi t / 2))-(\phi x / 2) \sin (\phi t / 2)+(\phi y / 2) \cos (\phi t / 2)$

$+x \cos (\phi t / 2) \cos (2 \phi t)+y \cos (\phi t / 2) \sin (2 \phi t)$

$+x \sin (2 \phi t) \sin (\phi t / 2)-y \cos (2 \phi t) \sin (\phi t / 2))$,

(6) $\quad F(t, x, y) \cdot \nabla \Lambda_{R}^{2}(t, x, y)=\frac{2}{R^{2}}(x \sin (\phi t / 2)-y \cos (\phi t / 2))$

$\times(-f(z)(-y \cos (\phi t / 2)+x \sin (\phi t / 2))+(\phi y / 2) \sin (\phi t / 2)+(\phi x / 2) \cos (\phi t / 2)$

$+x \sin (\phi t / 2) \cos (2 \phi t)+y \sin (\phi t / 2) \sin (2 \phi t)$

$-x \sin (2 \phi t) \cos (\phi t / 2)+y \cos (2 \phi t) \cos (\phi t / 2))$.

It is easy to check that $x^{2}+y^{2} \geq R^{2},|x| \leq R \sqrt{2}$ and $|y| \leq R \sqrt{2}$ if $(t, x, y) \in$ $\partial L_{R}$ for some $t$. Let $R$ be so large that

$$
\inf _{\partial L_{R}} f>10 .
$$

If $(t, x, y) \in L_{R}^{-}$, then $|x \cos (\phi t / 2)+y \sin (\phi t / 2)|=R$ and, by (5),

$$
F(t, x, y) \cdot \nabla \Lambda_{R}^{1}(t, x, y) \geq 2 f(z)-2 \phi \sqrt{2}-8 \sqrt{2}>0
$$

for sufficiently small $\phi \leq 1 /(2 \sqrt{2})$, hence (3) follows. In the same manner we see that if $(t, x, y) \in L_{R}^{+}$, then $|x \sin (\phi t / 2)-y \cos (\phi t / 2)|=R$ and, by $(6)$,

$$
F(t, x, y) \cdot \nabla \Lambda_{R}^{2}(t, x, y) \leq-2 f(z)+2 \phi \sqrt{2}+8 \sqrt{2}<0
$$

for small $\phi$, so (4) is also satisfied.

For $r>0$ we put

$$
\begin{aligned}
& \Xi_{r}^{1}(t, x, y)=\frac{1}{r^{2}}(x \cos (\phi t)+y \sin (\phi t))^{2}-1, \\
& \Xi_{r}^{2}(t, x, y)=\frac{1}{r^{2}}(x \sin (\phi t)-y \cos (\phi t))^{2}-1,
\end{aligned}
$$


and

$$
\begin{aligned}
K_{r} & =\left\{(t, x, y) \in \mathbb{R}^{3}: \Xi_{r}^{i}(t, x, y) \leq 0, i=1,2\right\}, \\
K_{r}^{-} & =\left\{(t, x, y) \in \mathbb{R}^{3}: \Xi_{r}^{1}(t, x, y)=0, \Xi_{r}^{2}(t, x, y) \leq 0\right\}, \\
K_{r}^{+} & =\left\{(t, x, y) \in \mathbb{R}^{3}: \Xi_{r}^{1}(t, x, y) \leq 0, \Xi_{r}^{2}(t, x, y)=0\right\} .
\end{aligned}
$$

Lemma 2. For $\phi>0, r>0$ sufficiently small,

$$
\begin{array}{ll}
F(t, x, y) \cdot \nabla \Xi_{r}^{1}(t, x, y)>0 & \left((t, x, y) \in K_{r}^{-}\right), \\
F(t, x, y) \cdot \nabla \Xi_{r}^{2}(t, x, y)<0 & \left((t, x, y) \in K_{r}^{+}\right) .
\end{array}
$$

Proof. A direct calculation shows that

(9) $\quad F(t, x, y) \cdot \nabla \Xi_{r}^{1}(t, x, y)=\frac{2}{r^{2}}(x \cos (\phi t / 2)+y \sin (\phi t / 2))$

$$
\times((x \cos (\phi t / 2)+y \sin (\phi t / 2))-\phi x \sin (\phi t)+\phi y \cos (\phi)
$$$$
+f(z)(x \cos (\phi) \cos (\phi t)+y \cos (\phi) \sin (\phi t)
$$

$$
\begin{aligned}
& +x \sin (\phi t) \sin (\phi t)-y \cos (\phi t) \sin (\phi t))), \\
F(t, x, y) \cdot \nabla & \Xi_{r}^{2}(t, x, y)=\frac{2}{r^{2}}(x \sin (\phi t / 2)-y \cos (\phi t / 2)) \\
& \times(-(-y \cos (\phi t)+x \sin (\phi t))+\phi y \sin (\phi t)+\phi x \cos (\phi t) \\
& +f(z)(x \sin (\phi t) \cos (\phi t)+y \sin (\phi t) \sin (\phi t) \\
& -x \sin (\phi t) \cos (\phi t)+y \cos (\phi t) \cos (\phi t))) .
\end{aligned}
$$

It is easy to check that $x^{2}+y^{2} \geq r^{2},|x| \leq r \sqrt{2}$ and $|y| \leq r \sqrt{2}$ if $(t, x, y) \in$ $\partial K_{r}$ for some $t$. Let $r$ be so small that

$$
\max _{\partial K_{r}} f<1 / 10 \text {. }
$$

If $(t, x, y) \in K_{r}^{-}$, then $|x \cos (\phi t)+y \sin (\phi t)|=r$ and, by (9),

$$
F(t, x, y) \cdot \nabla \Xi_{r}^{1}(t, x, y) \geq 2-4 \phi \sqrt{2}-8 \sqrt{2} f(z)>0
$$

for sufficiently small $\phi$, hence (7) follows. In the same manner we see that if $(t, x, y) \in K_{r}^{+}$, then $|x \sin (\phi t)-y \cos (\phi t)|=r$ and, by (10),

$$
F(t, x, y) \cdot \nabla \Xi_{r}^{2}(t, x, y) \leq-2+4 \phi \sqrt{2}+8 \sqrt{2} f(z)<0
$$

for small $\phi$, so (8) is also satisfied.

Let $\omega>0$ be another parameter. For $t<R / \omega$ put

$$
\begin{aligned}
& \Pi_{R, \omega}^{1}(t, x, y)=\frac{1}{(R-\omega t)^{2}}(x \cos (\phi t)+y \sin (\phi t))^{2}-1, \\
& \Pi_{R, \omega}^{2}(t, x, y)=\frac{1}{(R-\omega t)^{2}}(x \sin (\phi t)-y \cos (\phi t))^{2}-1,
\end{aligned}
$$

and

$$
P_{r, R, \omega}=\left\{(t, x, y) \in[0,(R-r) / \omega] \times \mathbb{R}^{2}: \Pi_{R, \omega}^{i}(t, x, y) \leq 0, i=1,2\right\},
$$




$$
\begin{aligned}
P_{r, R, \omega}^{-}=\left\{(t, x, y) \in[0,(R-r) / \omega] \times \mathbb{R}^{2}:\right. \\
\left.\Pi_{R, \omega}^{1}(t, x, y)=0, \Pi_{R, \omega}^{2}(t, x, y) \leq 0\right\}, \\
P_{r, R, \omega}^{+}=\left\{(t, x, y) \in[0,(R-r) / \omega] \times \mathbb{R}^{2}:\right. \\
\left.\Pi_{R, \omega}^{1}(t, x, y) \leq 0, \Pi_{R, \omega}^{2}(t, x, y)=0\right\} .
\end{aligned}
$$

Lemma 3. If $\phi$ and $\omega \leq r / 2$ are sufficiently small then

$$
\begin{array}{ll}
F(t, x, y) \cdot \nabla \Pi_{R, \omega}^{1}(t, x, y)>0 & \left((t, x, y) \in P_{r, R, \omega}^{-}\right), \\
F(t, x, y) \cdot \nabla \Pi_{R, \omega}^{2}(t, x, y)<0 & \left((t, x, y) \in P_{r, R, \omega}^{+}\right) .
\end{array}
$$

Proof. We have

$$
\begin{aligned}
& F(t, x, y) \cdot \nabla \Pi_{R, \omega}^{1}(t, x, y)=\frac{2(x \cos (\phi t)+y \sin (\phi t))}{(R-\omega t)^{3}} \\
& \times((-\phi x \sin (\phi t)+\phi y \cos (\phi t))(R-\omega t)+(x \cos (\phi t)+y \sin (\phi t)) \omega) \\
&+ \frac{2(x \cos (\phi t)+y \sin (\phi t))}{(R-\omega t)^{2}}(x f(z)+(x \cos (\phi t)+y \sin (\phi t))), \\
& F(t, x, y) \cdot \nabla \Pi_{R, \omega}^{2}(t, x, y)=\frac{2(x \sin (\phi t)-y \cos (\phi t))}{(R-\omega t)^{3}} \\
& \quad \times((\phi x \cos (\phi t)+\phi y \sin (\phi t))(R-\omega t)+(x \sin (\phi t)-y \cos (\phi t)) \omega) \\
& \quad+\frac{2(x \sin (\phi t)-y \cos (\phi t))}{(R-\omega t)^{2}}(y f(z)-(x \sin (\phi t)-y \cos (\phi t))) .
\end{aligned}
$$

Let $(t, x, y) \in P_{R, \omega}^{-}$, hence $|x \cos (\phi t)+y \sin (\phi t)|=R-\omega t$. Because $\cos (\phi t)$ $>0$ for small $\phi$ and $t \in[0,(R-r) / \omega]$, it follows by (13) that

$$
\begin{aligned}
F(t, x, y) \cdot \nabla \Pi_{R, \omega}^{1} & (t, x, y) \\
& \geq \frac{2 \omega}{R-\omega t}+2-2 \phi+\frac{2 x f(z)(x \cos (\phi t)+y \sin (\phi t))}{(R-\omega t)^{2}} \\
& \geq 2-2 \phi+\frac{2 x y f(z) \sin (\phi t)}{(R-\omega t)^{2}} \\
& \geq 2-2 \phi-4 \phi t f(z) \geq 2-2 \phi-4 \phi \frac{R-r}{\omega} f(z)>0
\end{aligned}
$$

for sufficiently small $\phi$. By a similar argument we conclude that if $(t, x, y) \in$ $P_{R, \omega}^{+}$then

$$
\begin{aligned}
F(t, x, y) \cdot \nabla \Pi_{R, \omega}^{2}(t, x, y) & \leq-2+\frac{2 \omega}{r}+\frac{2 y f(z)(x \sin (\phi t)-y \cos (\phi t))}{(R-\omega t)^{2}}+2 \phi \\
& \leq-2+1+\frac{2 x y \sin (\phi t) f(z)}{(R-\omega t)^{2}}+2 \phi \\
& \leq-1+2 \phi+2 \phi \frac{R-r}{\omega} f(z)<0,
\end{aligned}
$$

for sufficiently small $\phi$, so the proof of Lemma 3 is finished. 
The next family of functions and sets is obtained from the previous one by symmetry with respect to the hyperplane $\left\{(t, x, y) \in \mathbb{R}^{3}: t=\pi / \phi\right\}$, i.e.

$$
\begin{gathered}
\Sigma_{R, \omega}^{1}(t, x, y)=\Pi_{R, \omega}^{1}\left(\frac{2 \pi}{\phi}-t, x, y\right) \\
=\frac{1}{(\omega t+R-2 \pi(\omega / \phi))^{2}}(x \cos (\phi t)+y \sin (\phi t))^{2}-1, \\
\Sigma_{R, \omega}^{2}(t, x, y)=\Pi_{R, \omega}^{2}\left(\frac{2 \pi}{\phi}-t, x, y\right) \\
=\frac{1}{(\omega t+R-2 \pi(\omega / \phi))^{2}}(x \sin (\phi t)-y \cos (\phi t))^{2}-1, \\
S_{r, R, \omega}=\left\{(t, x, y) \in\left[\frac{2 \pi}{\phi}-\frac{R-r}{\omega}, \frac{2 \pi}{\phi}\right] \times \mathbb{R}^{2}: \Sigma_{R, \omega}^{i}(t, x, y) \leq 0, i=1,2\right\}, \\
S_{r, R, \omega}^{-}=\left\{(t, x, y) \in\left[\frac{2 \pi}{\phi}-\frac{R-r}{\omega}, \frac{2 \pi}{\phi}\right] \times \mathbb{R}^{2}:\right. \\
\left.\Sigma_{R, \omega}^{1}(t, x, y)=0, \Sigma_{R, \omega}^{2}(t, x, y) \leq 0\right\}, \\
S_{r, R, \omega}^{+}=\left\{(t, x, y) \in\left[\frac{2 \pi}{\phi}-\frac{R-r}{\omega}, \frac{2 \pi}{\phi}\right] \times \mathbb{R}^{2}:\right. \\
\left.\Sigma_{R, \omega}^{1}(t, x, y) \leq 0, \Sigma_{R, \omega}^{2}(t, x, y)=0\right\} .
\end{gathered}
$$

LEMMA 4. Under the assumptions of Lemma 3 we have

$$
\begin{array}{ll}
F(t, x, y) \cdot \nabla \Sigma_{R, \omega}^{1}(t, x, y)>0 & \left((t, x, y) \in S_{r, R, \omega}^{-}\right), \\
F(t, x, y) \cdot \nabla \Sigma_{R, \omega}^{2}(t, x, y)<0 & \left((t, x, y) \in S_{r, R, \omega}^{+}\right) .
\end{array}
$$

Proof. By symmetry, the proof of Lemma 3, up to inessential changes, also applies to Lemma 4.

Lemma 5. If $\phi<\omega /(4 \sqrt{2} R)$ then $P_{r, R, \omega} \subset L_{R}$ and $S_{r, R, \omega} \subset L_{R}$.

P r o of. Again by symmetry, it suffices to prove that $P_{r, R, \omega} \subset L_{R}$. If $(t, x, y) \in P_{r, R, \omega}$ then

$$
|x \cos (\phi t)+y \sin (\phi t)| \leq R-\omega t
$$

and

$$
|x \sin (\phi t)-y \cos (\phi t)| \leq R-\omega t
$$

SO

$$
|x \cos (\phi t)| \leq R-\omega t+|y \sin (\phi t)| .
$$

Because $1-|\sin (\phi t)| \leq|\cos (\phi t)|$, hence

$$
|x|(1-|\sin (\phi t)|) \leq R-\omega t+|y||\sin (\phi t)|
$$

and finally

$$
|x| \leq R-\omega t+(|x|+|y|)|\sin (\phi t)| \leq R-\omega t+2 \sqrt{2} R \phi t \leq R-\omega t / 2 .
$$


Similarly $|y| \leq R-\omega t / 2$, so we obtain

$$
|x \cos (\phi t / 2)+y \sin (\phi t / 2)| \leq(R-\omega t / 2)(|\sin (\phi t / 2)|+|\cos (\phi t / 2)|)
$$

and

$$
|x \sin (\phi t / 2)-y \cos (\phi t / 2)| \leq(R-\omega t / 2)(|\sin (\phi t / 2)|+|\cos (\phi t / 2)|) .
$$

We have

$$
|\sin (\phi t / 2)|+|\cos (\phi t / 2)| \leq \frac{\phi}{2} t+1 \leq \frac{\omega t}{2 R}+1 \leq \frac{\omega t}{2 R-\omega t}+1=\frac{R}{R-\omega t / 2},
$$

hence $|x \cos (\phi t / 2)+y \sin (\phi t / 2)| \leq R$ and $|x \sin (\phi t / 2)-y \cos (\phi t / 2)| \leq R$ and thus $(t, x, y) \in L_{R}$.

Proof of Theorem 1. Let $R, r, \omega$ and $\phi$ be such that the conclusions of the lemmas hold. Define

$$
\begin{aligned}
& Z=P_{r, R, \omega} \cup\left(K_{r} \cap\left(\left[\frac{R-r}{\omega}, \frac{2 \pi}{\phi}-\frac{R-r}{\omega}\right] \times \mathbb{R}^{2}\right)\right) \cup S_{r, R, \omega}, \\
& W=L_{R} \cap\left([0,2 \pi / \phi] \times \mathbb{R}^{2}\right) .
\end{aligned}
$$

Lemmas $1-4$ show that $U$ and $W$ are isolating segments over $[0,2 \pi / \phi]$,

$$
\begin{aligned}
Z^{-} & =P_{r, R, \omega}^{-} \cup\left(K_{r}^{-} \cap\left(\left[\frac{R-r}{\omega}, \frac{2 \pi}{\phi}-\frac{R-r}{\omega}\right] \times \mathbb{R}^{2}\right)\right) \cup S_{r, R, \omega}^{-}, \\
W^{-} & =L_{R}^{-} \cap\left([0,2 \pi / \phi] \times \mathbb{R}^{2}\right)
\end{aligned}
$$

are their exit sets, and

$$
\begin{gathered}
Z_{0}=W_{0}=\left\{(x, y) \in \mathbb{R}^{2}:|x| \leq R,|y| \leq R\right\}, \\
Z_{0}^{-}=W_{0}^{-}=\left\{(x, y) \in \mathbb{R}^{2}:|x|=R,|y| \leq R\right\} .
\end{gathered}
$$

Thus

$$
\mu_{W} \circ \mu_{W}=\operatorname{id}_{H\left(U_{0}, U_{0}^{-}\right)}, \quad \operatorname{Lef}\left(\mu_{W}\right)=1, \quad \mu_{Z}=\operatorname{id}_{H\left(Z_{0}, Z_{0}^{-}\right)} .
$$

Moreover, $Z \subset W$ because $K_{r} \subset L_{R}$ and Lemma 5 holds. Since $\chi\left(Z_{0}, Z_{0}^{-}\right)=$ -1 , all conditions (a), (b), and (c) are satisfied, hence Theorem 2 implies the result.

\section{References}

[Ma] J. Mawhin, Periodic solutions of some planar non-autonomous polynomial differential equations, J. Differential Integral Equations 7 (1994), 1055-1061.

[MMZ] R. Manásevich, J. Mawhin and F. Zanolin, Periodic solutions of complexvalued differential equations and systems with periodic coefficients, J. Differential Equations 126 (1996), 355-373.

[MM] K. Mischaikow and M. Mrozek, Chaos in Lorenz equations: a computer assisted proof, Bull. Amer. Math. Soc. 32 (1995), 66-72. 
[S] R. Srzednicki, Periodic and bounded solutions in blocks for time-periodic nonautonomous ordinary differential equations, Nonlinear Anal. 22 (1994), 707-737.

[S1] - On periodic solutions of planar differential equations with periodic coefficients, J. Differential Equations 114 (1994), 77-100.

[SW] R. Srzednicki and K. Wójcik, A geometric method for detecting chaotic dynamics, ibid. 135 (1997), 66-82.

[W] S. Wiggins, Global Bifurcation and Chaos. Analytical Methods, Springer, New York, 1988.

[W1] K. Wójcik, Isolating segments and symbolic dynamics, Nonlinear Anal. 33 (1998), 575-591.

[W2] —, On detecting periodic solutions and chaos in ODE's, ibid., to appear.

Institute of Mathematics

Jagiellonian University

Reymonta 4

30-059 Kraków, Poland

E-mail: wojcik@im.uj.edu.pl

Reçu par la Rédaction le 31.5.1999

Révisé le 20.10.1999 